ISSN: 2215-2644

revedu@gmail.com

Universidad de Costa Rica

Costa Rica

\title{
La retroalimentación evaluativa o feedback para los trabajos en grupo como estrategia de acción tutorial en la universidad
}

\author{
De la Torre-Laso, Jesús \\ La retroalimentación evaluativa o feedback para los trabajos en grupo como estrategia de acción tutorial en la \\ universidad \\ Revista Educación, vol. 43, núm. 1, 2019 \\ Universidad de Costa Rica, Costa Rica \\ Disponible en: http://www.redalyc.org/articulo.oa?id=44057415042 \\ DOI: https://doi.org/10.15517/revedu.v43i1.30062
}

Esta obra está bajo una Licencia Creative Commons Atribución-NoComercial-SinDerivar 3.0 Internacional. 
La retroalimentación evaluativa o feedback para los trabajos en grupo como estrategia de acción tutorial en la universidad

\section{Evaluative feedback for group work as a tutorial action strategy at universities}

Jesús De la Torre-Laso

Universidad de Salamanca. Departamento de Psicología

Social y Antropología, España

jesustl@usal.es

(iD http://orcid.org/0000-0002-9221-4027
DOI: https://doi.org/10.15517/revedu.v43i1.30062 Redalyc: http://www.redalyc.org/articulo.oa?id=44057415042

Recepción: 02 Agosto 2017

Aprobación: 05 Febrero 2019

\section{RESUMEN:}

La acción tutorial como proceso formativo se ha convertido en una exigencia del nuevo Espacio Europeo de Enseñanza Superior. En ese contexto, la retroalimentación entre el profesorado y el estudiantado pretende contribuir al modelo de enseñanza-aprendizaje. Los trabajos en grupo se han convertido, además, en una herramienta didáctica y su evaluación no suele conllevar un análisis cualitativo por parte del cuerpo docente. Este trabajo pretende indagar la percepción del alumnado de una asignatura del grado de Terapia Ocupacional de la Universidad de Salamanca, en España, sobre la utilidad de la tutoría de feedback o retroalimentación como estrategia de aprendizaje ante los trabajos en grupo. Para analizar este aspecto se ha realizado un estudio transversal con estudiantes que cursaron la asignatura en dos años consecutivos. Los resultados revelan la utilidad de dicha acción tutorial y muestran que la acción tutorial de retroalimentación es un método positivo para reportar información del aprendizaje. Se discuten las conclusiones y las limitaciones del estudio.

Palabras clave: Tutorías, Retroalimentación, Aprendizaje.

\section{Abstract:}

Tutorial action as part of educational development has become a requirement of the new European Higher Education Area (EHEA). Given this context, the intent of feedback between the faculty and students is to contribute to the teaching-learning model. Group work has also become another didactic tool which instructors do not generally assess through a qualitative analysis. This study probes at the perception of Occupational Therapy students who attended a required course at the University of Salamanca in Spain, with regards to tutoring feedback as a learning strategy for group work. In order to analyze this further, a cross-cutting study was conducted during two consecutive years with students enrolled in the course. The results revealed that using this type of tutorial action to provide feedback is a positive method for reporting on lessons learned. The conclusions and shortcomings of the study are also presented.

KEYWORDS: Tutorials, Feedback, Learning.

\section{INTRODUCCIÓN}

En el nuevo Espacio Europeo de Enseñanza Superior (EEES) se ha ido desarrollando una nueva forma de enseñar y aprender. En primer lugar, se ha optado por un sistema de aprendizaje autónomo y tutorizado, y ha dado lugar a un cambio de estrategia docente universitaria donde se pide que el personal docente desarrolle menos clases magistrales y se fomente el trabajo colaborativo. De esta forma, el profesorado se convierte en orientador, guía y evaluador de los procesos de aprendizaje. Es decir, destaca más su dimensión tutorial que su dimensión docente.

La acción tutorial se ha integrado en la acción docente, con el objeto de posibilitar, además, un proceso de retroalimentación con el estudiantado, y permitir así (en dicho proceso de enseñanza y aprendizaje) que conozcan sus errores de una manera directa, y se le faciliten alternativas para resolver sus dificultades, así como para motivarles, consolidar su aprendizaje y estimular su potencial (Gairín, Feixas, Guillamón y Quinquer, 2004). 
Desde hace varias décadas la tutoría académica, entendida como un elemento más de la práctica docente, ha ido alcanzando progresivamente un mayor interés en las instituciones universitarias, por lo que el cuerpo docente se convierte necesariamente, en una figura con función tutorial (Álvarez, 2013). Las actividades tutoriales tienen que concebirse como una parte de la formación integral la cual debe recibir el alumnado (Álvarez y López, 2013) donde el personal docente universitario utiliza su actividad docente para guiar y orientar al estudiantado en su itinerario, mientras que este construye su conocimiento y aprendizaje de manera autónoma. La tutoría académica adquiere, entonces, un papel muy importante en ese nuevo espacio formativo, un entorno en el cual reflexionar, hacer propuestas, interiorizar el aprendizaje, verificar su desarrollo y fomentar la adquisición de nuevas competencias.

En España, como en la mayoría de los países implicados en el nuevo EEES, la incorporación de la tutoría como actividad docente es relativamente reciente (Álvarez, García, Gil y Romero, 2000). Este modelo de intervención educativa forma parte de la estructura formativa que aúna clases magistrales con otras formas de docencia como los seminarios, las prácticas en el laboratorio, los estudios de caso, las prácticas de campo, comentarios de texto, simulación, las clases presenciales y los trabajos individuales y grupales.

En la actualidad coexisten tres grandes modelos de tutoría (Rodríguez, 2004) asociados a los principales modelos de Universidad:

- Modelo académico o docente: en esta manera de entender la tutoría, la acción docente se restringe a informar u orientar sobre su asignatura en un ambiente de máxima autonomía y libertad de todos los miembros de la comunidad universitaria. La tutoría es una forma más de metodología de enseñanza y una manera especial de intercambio de conocimientos. Puede materializarse en forma de explicación o comentario a los estudiantes sobre alguno de los puntos del programa o bien en forma de seminarios, ya sean programados o respondiendo a una necesidad coyuntural sobrevenida en el transcurso del desarrollo del programa.

Modelo de desarrollo personal: el objetivo que plantea es el desarrollo integral del estudiantado y por tanto la acción tutorial abarca ámbitos, lejos del estrictamente académico, para adentrarse en cuestiones profesionales y personales.

Modelo de desarrollo profesional: en este modelo, la tutoría en la Universidad se ve complementada por la tutoría fuera del marco universitario donde se desarrollan las prácticas en situaciones reales y en las cuales el principal objetivo es el desarrollo de destrezas y competencias personales, académicas y profesionales para que el perfil del alumnado se ajuste al máximo a los requerimientos del puesto profesional que se va a ver obligado a desempeñar.

Desde la perspectiva puramente académica y, entendiendo la tutoría como una modalidad de enseñanza en la que se ayuda de manera personalizada al estudiantado, se reconoce como una estrategia didáctica (Pérez, 2006), pues pretende ser un proceso de aprendizaje por sí misma y a la vez, se complementa con otras estrategias educativas, como las clases magistrales.

Las tutorías son de utilidad para el personal docente, además, le aportan información sobre el grado de dificultad que, para el estudiantado, entraña una determinada tarea o asignatura, y las partes del programa que pueden requerir cambios futuros. Igualmente se pueden emplear para tener información sobre el nivel de aprendizaje de los estudiantes, y son una herramienta de gran valor a la hora de evaluar (Juste y Carballo, 2010).

En el propósito del presente estudio, las tutorías de retroalimentación o feedback se encuadran dentro del ámbito de las tutorías académicas o docentes, como modelo de tutoría programada que trata de devolver información al alumnado. En este proceso, la información es la parte central del desarrollo formativo, pues el estudiantado recibe del profesorado información objetiva de sus avances y le permite situar su aprendizaje en el desarrollo curricular y extrapolarlo a futuras acciones. 


\section{MARCO TEÓRICO}

\section{La retroalimentación como estrategia de acción tutorial}

La retroalimentación o feedback es entendida como "cualquier información, que se proporciona a quien desempeña una acción cualquiera, respecto de ese desempeño" (Ross y Tronson, 2005, p. 173). Etimológicamente el feedback formativo o retroalimentación se puede entender o definir (Hattie y Timperley, 2007) como la "información proporcionada por un agente (por ejemplo, maestro, compañero, padre) en relación con los aspectos del rendimiento o la comprensión de uno" (p. 81). Según Shute (2008) es un componente esencial del proceso educativo y en este ámbito, la retroalimentación se refiere a aquella información que se comunica al estudiantado que tiene la intención de modificar el pensamiento o comportamiento con el propósito de mejorar el aprendizaje.

Ante estos planteamientos, tratar la retroalimentación dentro de un contexto de aprendizaje no es considerarlo como un fin en sí mismo, sino como un instrumento de utilidad para el personal docente y el alumnado en ese proceso de enseñanza-aprendizaje, donde el primero emite información al segundo sobre su proceso de aprendizaje con el objeto de facilitar los cambios de su itinerario formativo (Fornells, Juliá, Arnau y Martínez, 2008).

Este proceso de retroalimentación juega un papel importante en la autoevaluación del aprendizaje porque puede mostrar de manera directa las fortalezas y debilidades del aprendizaje del estudiantado y se ha demostrado que tiene un impacto significativo en su rendimiento (Rossiter, 2016). Para Ferguson (2011), el feedback es visto como una manera importante de la enseñanza y puede servir para facilitar el desarrollo de estudiantes independientes capaces de monitorear, evaluar y regular su propio aprendizaje, lo que les permite alimentarse en la práctica profesional. Incluso desde el punto de vista cada estudiante, recibir retroalimentación es percibido como un signo de calidad docente (Torre, Sebastián y Simpson, 2003).

La retroalimentación puede mejorar los procesos de aprendizaje y los resultados posteriores del estudiantado, aunque el efecto de este proceso de formación es eficaz si se realiza bajo condiciones controladas y que no interfieran o dificulten dicho proceso de formación. Entre los procesos eficaces de retroalimentación se sitúan aquellos que se centran en la información sobre la tarea y cómo mejorarla (Morales, 2012). Así, si la retroalimentación se centra en la autorregulación puede mejorar el compromiso con la tarea, y puede incidir positivamente en aspectos su capacidad para lograr la tarea y su voluntad de seguir trabajando en ella mientras que no es eficaz si se concentra en el o la estudiante como una persona (Hattie y Timperley, 2007).

Para que la acción tutorial sea eficaz, la persona tutora debe actuar con cierto rigor, siguiendo un proceso metodológico determinado. Sadler (2010) identificó tres factores clave para la retroalimentación efectiva: el estudiantado necesita una referencia en el concepto de la meta de aprendizaje, se debe comparar el desempeño actual con el objetivo y debe tomar medidas para reducir la diferencia entre el rendimiento real y el objetivo. La eficacia del feedback formativo o retroalimentación radica en cuanto que sirve para comunicar al alumnado información sobre el grado de adquisición de los objetivos y/o competencias, proporcionándole así, recursos que le permitan avanzar en este aprendizaje. Para que la retroalimentación se ajuste a los criterios establecidos inicialmente, cada estudiante debe conocer esos objetivos, pues serán el referente para entender cómo debe hacer sus tareas y los criterios por los cuales se les va a evaluar. Es lo que se ha denominado feedforward o mirar hacia adelante. Este proceso se puede producir en dos momentos (Morales, 2012), bien como una orientación previa sobre lo que se le pide al estudiantado en la dinámica de aprendizaje, en forma de orientación inicial, identificando las rúbricas para que conozca los objetivos del sistema de evaluación, a través de orientaciones o ejemplos sobre lo que se espera del alumnado, o bien, cuando la tarea ha finalizado para corregir sus deficiencias y mejorar el rendimiento futuro. Siguiendo esta consideración, el feedback formativo debería ser el de conseguir desarrollar un proceso de metacognición en cada estudiante con el objetivo de que pueda progresar y autorregular su proceso de aprendizaje. En este sentido, para Askew y Lodge (2000) 
"la función del feedback sería propiciar un proceso que estimule la autorreflexión y la reflexión de los otros sobre el mismo proceso de aprendizaje" (p. 3), pues la identificación que realizan los estudiantes ante una retroalimentación exitosa tiene que ver con un vínculo claro entre las tareas de evaluación y directrices, los marcos de evaluación, y la retroalimentación ofrecida (Ferguson, 2011).

No obstante, personas autoras han encontrado que el feedback formativo no es eficaz y no ayuda al estudiantado a superar esa brecha entre su rendimiento y el aprendizaje para posteriores acciones. En ocasiones esto se debe a la incapacidad del alumnado para aprovechar las oportunidades que ofrece la retroalimentación, en otras, la variable mediadora tiene que ver con el desarrollo cognitivo del estudiantado, sus estilos de aprendizaje (Vickerman, 2009), el estado emocional (Torre, 2007) o sus creencias sobre el aprendizaje y su proceso.

El planteamiento de este artículo parte de la idea de que entender que en el modelo educativo actual está plenamente aceptada la importancia de la retroalimentación en el proceso de evaluación (Gértrudix, Esteban, Hortal y Gálvez, 2016). La efectividad de dicha retroalimentación tiene un impacto diferente en estudiantes dependiendo del uso que desempeñen las metodologías. En ocasiones, al estudiantado solo le interesa la nota o el proceso está orientado a los aciertos y errores y en otras, el proceso de retroalimentación no aporta nada constructivo para ellos. La interacción puede ser aprovechada por el cuerpo docente para implementar estrategias que sirvan como espacios de aprendizaje y evaluación y, por ende, de retroalimentación.

La experiencia desarrollada se construye ante la necesidad de investigar la utilidad de los procesos de retroalimentación en el alumnado dentro de los apoyos de docencia de los que dispone el personal docente, entre los cuales se encuentra la acción tutorial. Un espacio donde esta se puede ejercer y, por tanto, darse retroalimentación educativa en los trabajos colaborativos o trabajo en grupo.

\section{Los trabajos en grupo}

Los trabajos en grupo son una modalidad de aprendizaje cada vez más utilizada en el EEES y suponen un método de trabajo cooperativo (cooperative learning) donde se pasa de la responsabilidad del estudiantado a una corresponsabilidad cooperativa en la cual se comparten estrategias, se alcanzan metas y se consigue una evaluación global.

El nuevo marco europeo de educación universitaria enfatiza el trabajo en grupo como una estrategia básica para la adquisición de competencias interpersonales, pues su desempeño favorece las destrezas sociales, la cooperación, la interacción y el trabajo en equipo (Gámez y Torres, 2013). En líneas generales, los trabajos en grupo se han constituido como una modalidad de enseñanza en el EEES (Sellés y Carril, 2012) donde se pretende fomentar el aprendizaje autónomo, el desarrollo de competencias intelectuales y sociales variadas, las capacidades de resolución de problemas y el desempeño de trabajos colaborativos (Domínguez, López y Cobos, 2012).

El ejercicio colaborativo se ha desarrollado como una competencia básica en la práctica profesional de cualquier titulado. Mediante esta dinámica, el estudiante debe interaccionar con otros para lograr unas metas compartidas y a través de la interacción, debe aclarar ideas y construir nuevas formas de pensamiento (Guillies y Boyle, 2010). Generalmente, la modalidad de trabajo en grupo se suele realizar dentro del aula y fuera de esta, con la presencia del profesorado o sin ella. El resultado de las tareas grupales cooperativas, los logros del estudiantado están relacionados entre sí, de tal forma que existe una relación positiva entre los objetivos alcanzados: un estudiante consigue su objetivo, solo si los otros integrantes del grupo alcanzan el suyo.

Considerando su funcionalidad, el trabajo en grupo pretende dos objetivos: a) aprender a trabajar de manera cooperativa, b) desarrollar un estudio, de manera consensuada, sobre un aspecto curricular concreto o una determinada línea de investigación.

En el modelo tradicional de enseñanza, donde no se incluían otras modalidades de enseñanza diferentes de la clase magistral unidireccional (docente-alumnado) la retroalimentación se reducía a menudo a la 
calificación después de una prueba objetiva, con un formato generalmente numérico. La evaluación de los trabajos en grupo requiere un proceso de retroalimentación que vaya más allá de la mera calificación, un contexto en el cual el estudiantado puede recibir información sobre el desarrollo de su trabajo y el logro de las competencias.

Partiendo de estos planteamientos, la experiencia del presente artículo pretende estudiar la relación que existe entre la acción tutorial con el ejercicio de retroalimentación que le puede ofrecer el personal docente al alumnado en los contextos de evaluación del trabajo en grupo. Así, el objetivo principal de la investigación es determinar la utilidad de la acción tutorial del estudiantado universitario después de finalizar una tarea de trabajo grupal.

\section{Procedimiento metodológico}

\section{Participantes}

La muestra estuvo constituida por 92 estudiantes que cursaron una clase de segundo curso del Grado de Terapia Ocupacional de la Universidad de Salamanca en dos años consecutivos ( 50 en el primer año y 42 en el segundo). Las personas participantes en el presente estudio fueron aquellas que acudieron a las tutorías de retroalimentación: 67 sujetos (el 72,83\% del alumnado matriculado el primer año y del 88,01\% en el segundo año) y participaron en la encuesta los 67 sujetos (el 100\% de quienes acudieron a dicha tutoría).

\section{Descripción y desarrollo del estudio}

El estudio se efectuó de manera transversal en dos años consecutivos con el estudiantado y tuvo cuatro etapas (Figura 1). En la primera etapa, de carácter informativo donde se comunicó a los estudiantes las fases del proceso de acción tutorial; la segunda etapa donde el alumnado elaboró el trabajo y lo expusieron en clase; la tercera etapa evaluativa consistió en el desarrollo de la acción tutorial y retroalimentación, y la cuarta etapa, de carácter evaluativo, donde se realizó el cuestionario elaborado ad hoc.

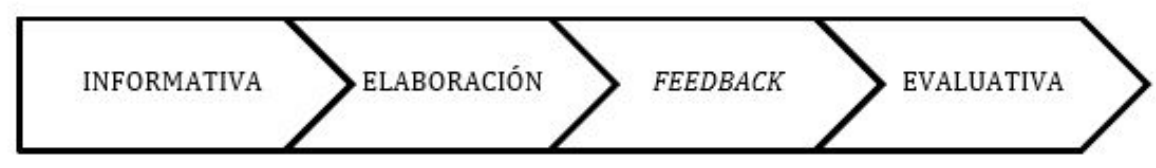

\section{FIGURA 1}

Fases del proyecto

Fuente: Elaboración propia

El procedimiento de formación tutorial siguió algunas de las recomendaciones para que el desarrollo de las tutorías formativas sea efectivo (Apodaca 2006; Morales, 2012 y Evans, 2013) y en concreto, el procedimiento seguido la explicación del espacio de acción tutorial y el desarrollo de la tutoría, emplearon las siguientes estrategias:

- Información previa. Antes de la iniciación del trabajo, se le explicaron al estudiantado los aspectos formales e informales que debería tener el trabajo, tales como la composición de los grupos, el tema a tratar, el desarrollo metodológico, el peso de la actividad en la evaluación final, etc. Con esta acción se pretendía que el alumnado tuviera una información previa de manera clara sobre los aspectos que requería la tarea. Incluyendo esta visión dentro del EEES donde el proceso de enseñar no se limita a transmitir conocimientos, sino que se entiende como el proceso donde cada docente muestra 
contenidos educativos, donde se le enseña al estudiantado a aprender, se potencian sus capacidades, por lo cual resulta fundamental que se disponga de una información inicial para mirar hacia adelante, proceso denominado feedforward. Así, se prepara al estudiante para resolver los aspectos de la tarea con éxito.

Rúbricas. Los criterios de evaluación por los que el alumnado va a ser evaluado es una de las claves dentro de cualquier diseño de una adecuada metodología de evaluación. Las rúbricas (Martínez y Raposo, 2010) favorecen el proceso de enseñanza-aprendizaje porque actúa de guía, y también la evaluación, porque permite objetivar cualquier trabajo del alumnado y restarle el componente de subjetividad que pudiera tener quien evalúa. En el presente trabajo, las rúbricas se utilizaron como punto de partida para efectuar las calificaciones posteriores, guiar el aprendizaje del estudiantado y servir como sistema autoevaluativo.

Exposición de trabajos modelo. Los trabajos modelo sirven para que el alumnado tenga ejemplos prácticos, no solo de otras actividades realizadas por estudiantes anteriores, sino que sirvan como referencia de los elementos positivos o negativos, de cara a optimizar su aprendizaje. El estudiantado valora la utilidad de este enfoque para atender a otras estructuras y diseños (Handley y Williams, 2011).

Tutoría de retroalimentación. Al finalizar el trabajo propuesto y después de la jornada de presentación, se volvió a reunir al alumnado que, de manera voluntaria, quisiera conocer los efectos del trabajo, las explicaciones sobre el contenido y la calidad de este, sus fortalezas y debilidades, atendiendo a las rúbricas establecidas.

Cuestionario. Dos semanas de la tutoría cada estudiante rellena el cuestionario elaborado al efecto.

\section{Instrumentos}

El cuestionario de satisfacción de la tutoría de retroalimentación fue elaborado ad hoc para medir el grado de bienestar, conformidad o satisfacción con el proceso de tutoría y está basada en los elementos fundamentales de estos procesos formativos.

El cuestionario está formado por 16 preguntas: 14 preguntas trataban sobre los componentes de la tutoría, y cada participante debía indicar su grado de acuerdo con cada afirmación utilizando una escala tipo Likert con cuatro opciones de respuesta (desde 0 completamente en desacuerdo, hasta 4 completamente de acuerdo). De las otras dos preguntas restantes, una solicitaba una valoración general, en una escala de 0 a 10 y otra le requería que expresara de forma abierta sus impresiones sobre la tutoría. Las dimensiones que se utilizaron se resumen en la Tabla 1

TABLA 1

\section{Dimensiones del instrumento de medida}

\begin{tabular}{ll}
\hline DIMENSIONES & ÍTEMS \\
\hline Aportaciones docentes & 3 ítems \\
Utilidad de la tutoría & 4 ítems \\
Metodologia utilizada & 3 ítems \\
Conformidad con la nota & 2 items \\
Evaluación general & 2 items \\
\hline
\end{tabular}

Fuente: Elaboración propia

Los resultados obtenidos en el presente estudio han avalado su fiabilidad ( $\alpha$ de Cronbach $=0,75$ ), por encima del criterio 0,70 recomendado para la consistencia de los resultados (Nunnally, 1987). 


\section{ANÁLISIS Y DISCUSIÓN DE LOS RESULTADOS}

Para la mayoría de estudiantes la tutoría de retroalimentación ha sido útil y ninguno de los sujetos valoró de manera negativa la acción tutorial. En líneas generales, todos los ítems planteados presentan respuestas positivas (Tabla 2).

El análisis transversal de la acción tutorial efectuado durante los dos años consecutivos del estudio efectuado ha mostrado resultados de satisfacción similares, lo que permite concluir valorar de manera positiva este espacio de retroalimentación como efectivo. Así mismo, el hecho de que el 100\% de estudiantes que participaron en la acción tutorial fueran sujetos del estudio permite valorar de manera fiable los resultados de este.

La utilidad de la tutoría de retroalimentación ha sido el ítem señalado con una mayor valoración por parte del estudiantado. En concreto, el 85,07\% de ellos está de acuerdo o completamente de acuerdo en señalar como provechosa esta acción formativa. En este sentido, la acción tutorial es un elemento de calidad de la actividad docente y un requisito para dar respuesta a las demandas del alumnado, tal y como muestran otros autores (Álvarez, 2008).

TABLA 2

Puntuaciones de la dimensión utilidad de la tutoría

\begin{tabular}{lllll}
\hline ÍTEMS & Mín. & Max. & $\begin{array}{l}\text { Media } \\
\text { (Total) }\end{array}$ & $\begin{array}{l}\text { D. T. } \\
\text { (Total) }\end{array}$ \\
\hline $\begin{array}{l}\text { El planteamiento ha sido útil } \\
\begin{array}{l}\text { Los contenidos han satisfecho mis } \\
\text { necesidades }\end{array}\end{array}$ & 1 & 4 & 3,34 & 0,78 \\
$\begin{array}{l}\text { Ha ayudado a realizar mejores } \\
\text { trabajos. }\end{array}$ & 1 & 4 & 2,49 & 0,78 \\
.- & & 4 & 3,24 & 0,81 \\
\end{tabular}

Fuente: Elaboración propia

Nota: $0=$ Completamente en desacuerdo; $4=$ Completamente de acuerdo.

En cuanto a la metodología empleada en la tutoría, el 80,3\% de los sujetos está de acuerdo o muy de acuerdo con el tiempo dedicado a esta (para cada grupo se dispuso de, aproximadamente, 15 o 20 minutos de tutoría de retroalimentación). Un 81,8\% considera adecuado utilizar de manera visual el trabajo para corregirlo y aportar la devolución de la información, y el 54\% mantiene una postura intermedia (ni de acuerdo/ni desacuerdo) con el tiempo empleado para las explicaciones (Tabla 3).

En cuanto a la retroalimentación aportada, el 74,63\% de personas encuestadas considera que las explicaciones han sido claras, concretas y fáciles de entender, y las críticas mostradas han sido constructivas (un 73,13\% está de acuerdo o completamente de acuerdo con esta afirmación).

TABLA 3

Medias y desviaciones típicas de la dimensión Aportaciones

\begin{tabular}{lllll}
\hline ÍTEMS & Media (Año 1) & D. T. (Año 1) & Media (Año 2) & D. T. (Año 2) \\
\hline $\begin{array}{l}\text { Explicaciones } \\
\text { Claras }\end{array}$ & 3,03 & 0,85 & 2,95 & 0,88 \\
$\begin{array}{l}\text { Críticas } \\
\begin{array}{l}\text { Constructivas } \\
\text { Información }\end{array}\end{array}$ & 3,10 & 0,80 & 2,86 & 0,92 \\
& 3.30 & 0.65 & 3.05 & 0.70
\end{tabular}

Fuente: Elaboración propia 
La mayoría de los sujetos que participaron en el estudio (el 65,67\%) está de acuerdo o completamente de acuerdo con el proceso de retroalimentación, sobre todo con las exposiciones de los trabajos modelo, en los momentos previos al desarrollo de la tarea.

Así mismo, la utilidad de la tutoría se ve expuesta cuando se le pregunta al estudiantado sobre las informaciones que se les muestran. El 83,58\% expresa estar de acuerdo o totalmente de acuerdo con que la tutoría de retroalimentación aporta información sobre los detalles del trabajo expuesto.

En cuanto a las perspectivas de futuro y a la generalización del proceso de tutorías, el $76,12 \%$ considera que es una metodología docente adecuada. El 89,55\% manifestó que la tutoría de evaluación se podría exportar a otras asignaturas y, por lo tanto, que esta estrategia propuesta podría tener un papel fundamental en la metodología formativa de otras asignaturas, en consonancia con lo que sostienen otros estudios (Gil, Martínez, Tunnicliffe, y Moneo, 2013).

La acción tutorial de retroalimentación se utilizó, además, para devolver al estudiantado la calificación numérica (la nota) del trabajo realizado. Los estudiantes valoraron de manera explícita tener información que correlacionara la calificación y los comentarios aportados. En este sentido, aproximadamente la mitad de ellos (el 46,87\%) se encontraba justa la nota en función de las informaciones aportadas por el personal docente.

La valoración global de la acción tutorial reflejó unas puntaciones elevadas. El primer año, puntuaron la tutoría con un 7,43 (sobre 10 puntos), mientras que el segundo año la puntuación alcanzó 7,31 puntos.

\section{Conclusiones}

El trabajo se ha centrado en estudiar la utilidad de la retroalimentación en los procesos de acción tutorial, como estrategia de comunicación y proceso de aprendizaje ante los trabajos que desarrolla el estudiantado de manera grupal.

La actividad de retroalimentación que se ha desarrollado forma parte del camino de ida y vuelta que se promueve en el EEES. Se ha considerado que las tutorías realizadas por el personal docente, y en particular, la tutoría de retroalimentación como una tarea de interacción para desarrollar las competencias en el alumnado de cara a su desarrollo académico y profesional (Boud y Molloy, 2013).

El estudio refleja que la acción tutorial después de finalizar una tarea de trabajo grupal es percibida por el estudiantado universitario como un contexto de evaluación útil. La acción tutorial sirve como un proceso de retroalimentación de la información de forma unidireccional (docente-alumnado) de tal manera que las personas participantes perciben que se les aporta información sobre los trabajos efectuados, sus fortalezas y debilidades, en consonancia con otros estudios que han encontrado que la retroalimentación es una estrategia útil para mejorar el rendimiento académico del estudiantado (Hilli, Melender, Salmu y Jonsén, 2014). Este planteamiento podría servir para líneas de investigación futura que podrían dirigirse a valorar bidireccionalmente el contenido de la información que se muestra en dichas tutorías.

Los resultados concluyen que la acción tutorial se puede convertir en un recurso de retroalimentación eficaz. Puede proporcionar una entrada de información, persigue contribuir a la resolución de problemas, potenciar la confianza del estudiante hacia su tutor, motivar en su formación académica y, por ende, facilitar el éxito en su rendimiento.

La puesta en marcha de tutorías sobre las actividades del alumnado contribuye a generar una mayor flexibilidad en el proceso de aprendizaje-enseñanza. Estas tutorías de retroalimentación son una metodología docente adecuada, pues no solo puede aportar una información valiosa acerca de sus resultados, sino que también permite reflexionar sobre las experiencias de su propio aprendizaje, en consonancia con las conclusiones de otros estudios (Paoloni y Rinaudo, 2013). En este sentido, la acción tutorial ha ayudado a madurar el compromiso del estudiantado, la experiencia personal de aprendizaje y el desarrollo de la autoeficacia. 
La metodología aplicada desde el punto de vista formal (el tiempo empleado y la exposición visual) han sido valoradas por estudiantes de manera satisfactoria porque les permitía entender los contenidos y lo que se esperaba de ellos. En la evaluación de los trabajos en grupo, la acción tutorial de retroalimentación puede ser un espacio de interacción proactivo que ofrece información sobre los avances de su aprendizaje o servir de asesoramiento sobre las partes del programa en ejecución. Así mismo, le permite al estudiantado reflexionar, hacer propuestas, interiorizar el aprendizaje, verificar su desarrollo y fomentar la adquisición de nuevas competencias.

Este estudio presenta algunas limitaciones. La primera tiene que ver con las respuestas, ya que trabajar con opiniones siempre supone una visión subjetiva, con percepciones muy distintas y, a veces, contradictorias ante la misma realidad. Así mismo, se ha necesitado tener otras referencias comparativas con estudios similares sobre la retroalimentación de los trabajos en grupo para comparar los resultados.

En sucesivas investigaciones, sería necesario estudiar otro tipo de efectos que pueden influir a la hora de estudiar la eficacia de las tutorías de retroalimentación, como el contenido del feedforward en la orientación previa, la posibilidad de proporcionar a cada estudiante una segunda oportunidad para poder efectuar un trabajo de mayor calidad, el desarrollo de lo expuesto en la jornada de tutoría, o la necesidad o no de poder establecer un diálogo más amplio, con mayor interacción y participación del alumnado. En definitiva, desarrollar una retroalimentación formativa eficaz, de mayor calidad que contribuya al crecimiento personal y profesional del estudiantado.

\section{REFERENCIAS}

Álvarez, M. (2008). La tutoría académica en el Espacio Europeo de Educación Superior. Revista Interuniversitaria de Formación del Profesorado, 22(1), $71 \# 88$.

Álvarez, P. R. (2013). La función tutorial del profesorado universitario: Una nueva competencia de la labor docente en el contexto del EEES. Revista portuguesa de pedagogía, 47(2), 85-106. http://dx.doi.org/10.14195/1647-86 $14 \_47-2 \_5$

Álvarez, P. R. y López, D. (2013). Tutoría académica personalizada para estudiantes universitarios deportistas de alto nivel. Revista Brasileira de Orientação Profissional, 14(2), 239-253.

Álvarez, V., García, E., Gil, J. y Romero, S. (2000). La orientación en la Universidad en el contexto de una docencia de calidad. En J. Ruiz y A. Medina (Coords.), Orientación educativa e Intervención Psicopedagógica, I Jornadas Andaluzas de Orientación y Psicopedagogía (pp. 209-252). Jaén, España: Servicio de Publicaciones de la Universidad de Jaén.

Apodaca, P. (2006). Estudio y trabajo en grupo: el aprendizaje cooperativo. En M. de Miguel (Dir.), Metodologias de enseñanza y aprendizaje para el desarrollo de competencias: Orientaciones para el profesorado universitario ante el espacio europeo de educación superior. Madrid: Alianza Editorial.

Askew, S. y Lodge, C. (2000). Gifts, ping pong and loopslinking feedback and learning. En S. Askew (Ed.), Feedback for learning (pp. 1-18). Londres: Routledge Palmer.

Boud, D. y Molloy, E. (2013). Rethinking models of feedback for learning: the challenge of design. Assessment $y$ evaluation in higher education, 38(6), 698-712.

Domínguez, G., López, A. y Cobos, D. (2012). La tutoría como valor añadido de la práctica docente. Una experiencia de Innovación Universitaria en el marco burocrático de Bolonia. @ Tic. Revista d'innovacio educativa de la Universitat de Valencia, 9, 61-71.

Evans, C. (2013). Making Sense of Assessment Feedback in Higher Education. Review of Educational Research, 83(1), 70-120. https://doi.org/10.3102/0034654312474350

Ferguson, P. (2011). Student perceptions of quality feedback in teacher education. Assessment y Evaluation in Higher Education, 36(1), 51-62. http://dx.doi.org/10.1080/02602930903197883 
Fornells, J. M., Juliá, X., Arnau, J. y Martínez, J.M. (2008). Feedback en educación médica. Educación Médica, 11(1), 7-12. Recuperado de http://scielo.isciii.es/pdf/edu/v1 $\ln 1 /$ formacion.pdf

Gairín, J., Feixas, M., Guillamón, C. y Quinquer, D. (2004) La tutoría académica en el escenario europeo de la Educación Superior. Revista Interuniversitaria de Formación del Profesorado, 18(1), 61-77.

Gámez, M. J. y Torres, C. (2013). Las técnicas de grupo como estrategia metodológica en la adquisición de la competencia de trabajo en equipo de los alumnados universitarios. Journal for Educators, Teachers and Trainers, $4(1), 14-25$.

Gértrudix, M., Esteban, N., Hortal, E. y Gálvez, M. C. (2016). La transformación necesaria, en la innovación docente con TIC como instrumento de transformación. Madrid: Dyckinson.

Gil, A., Martínez, A., Tunnicliffe, A. y Moneo, J.M. (2013). Estudiantes universitarios y calidad del Plan de acción tutorial. Valoraciones y mejoras. REDU. Revista de Docencia Universitaria, 11(2), 63-87. http://doi.org/10.49 95/redu.2013.5567

Guillies, R. M. y Boyle, M. (2010). Teachers' reflections on cooperative learning: Issues of implementation. Teaching and Teacher Education, 26(4), 933-940. http://doi.org/10.1016/j.tate.2009.10.034

Handley, K. y Williams, L. (2011). From copying to learning: using exemplars to engage students with assessment criteria and feedback. Assessment y Evaluation in Higher Education, 36(1), 95-108.

Hattie, J. y Timperley, H. (2007). The Power of Feedback. Review of Educational Research, 77(1), 81-112. http://d oi.org/10.3102/003465430298487

Hilli, Y., Melender, H. L., Salmu, M. y Jonsén, E. (2014). Being a preceptor-A Nordic qualitative study. Nurse education today, 34(12), 1420-1424.

Juste, M. R. P., y Carballo, J. S. (2010). Ventajas e inconvenientes de la tutoría grupal como estrategia docente: estudio de caso. Bordón. Revista de pedagogía, 62(1), 155-166.

Martínez, M. E. y Raposo, M. (2010). Seguimiento de trabajos tutelados en grupo mediante rúbricas. En Vicerrectoría de Formación e Innovación Educativa, La docencia en el nuevo escenario del EEES (pp. 567-570). Vigo, España: Universidad.

Morales, P. (2012). La información de retorno en la evaluación (feedback). En J. C. Torre Puente, Educación y nuevas sociedades: la formación inicial del docenteado de infantil y primaria. Madrid: Universidad Pontificia de Comillas.

Nunnally, J. C. (1987). Teoría Psicométrica. México: Editorial Trillas.

Paoloni, P. V.y Rinaudo, M. C. (2013). Los procesos de feedback desde una perspectiva multidimensional. Un estudio orientado a promover autorregulación en estudiantes universitarios. En P. Paoloni, M. C. Rinaudo y A. González Fernández (Eds.), Cuestiones en Psicología Educacional. Perspectivas teóricas, metodológicas y estudios de campo. Tenerife, Islas Canarias: Editorial Sociedad Latinoamericana de Comunicación Social (SLCS).

Pérez, A. (2006). Tutorías. En M. de Miguel (Coord.) Metodologías de enseñanza y aprendizaje para el desarrollo de competencias (pp. 133-167). Madrid: Alianza Editorial.

Rodríguez, S. (2004). Manual de Tutoría Universitaria. Recursos para la acción. Barcelona: Octaedro.

Rossiter,J. A. (2016). Using an understanding of feedback processes to improve student learning. IFAC-PapersOnLine, 49(6), 57-62.

Ross, P. y Tronson, D. (2005). Providing quality feedback - Where to from here? UniServe Science Blended Learning Symposium Proceedings. Recuperado de https://bit.ly/2sC5INn

Sadler, D. R. (2010). Beyond feedback: developing student capability in complex appraisal. Assessment y Evaluation in Higher Education, 35(5), 535-550.

Sellés, N. H., y Carril, P. C. M. (2012). Trabajo colaborativo en entornos e-learning y desarrollo de competencias transversales de trabajo en equipo: Análisis del caso del Máster en gestión de Proyectos en Cooperación Internacional, CSEU La Salle. REDU. Revista de Docencia Universitaria, 10(2), 411-434.

Shute, V. J. (2008). Focus on Formative Feedback. Review of Educational Research, 78(1), 153-189. http://doi.org/ $10.3102 / 0034654307313795$ 
Torre, D. M., Sebastian, J. L. y Simpson, D. E. (2003). Learning activities and high-quality teaching: perceptions of third-year IM clerkship students. Academic Medicine, 78(8), 812-814.

Torre, J. C. (2007). Una triple alianza para un aprendizaje universitario de calidad. Madrid: Universidad Pontificia Comillas.

Vickerman, P. (2009). Student perspectives on formative peer assessment: an attempt to deepen learning? Assessment y Evaluation in Higher Education, 34(2), 221-230. http://doi.org/10.1080/02602930801955986

\section{BY-NC-ND}

